\title{
The Combination of Xanafide and Cytarabine Has Unique Activity in Acute Myeloid Leukaemia
}

\author{
a report by \\ Harry P Erba \\ Associate Professor, Division of Hematology/Oncology, University of Michigan Health Systems
}

DOI: $10.17925 / E O H \cdot 2007 \cdot 0.2 .30$

Secondary acute myeloid leukaemia (SAML) evolves from a prior myelodysplastic syndrome (MDS) or myeloproliferative disorder (MPD) or occurs due to prior exposure to leukaemogenic therapy - also called treatment-related AML (tAML). ${ }^{1}$ Patients with SAML have an extremely poor prognosis, with only approximately $25 \%$ achieving remission following therapy with the standard regimen of bolus daunorubicin for three days and continuous-infusion cytarabine for seven days, the so-called ' $3+7$ ' regimen. ${ }^{2}$ The probability of achieving complete remission (CR) is affected by both patient- and diseaserelated factors. ${ }^{3}$ Important patient-related factors include age, associated co-morbid diseases and major organ dysfunction. Diseaserelated factors present in the leukaemia cells include specific numerical and structural chromosomal (cytogenetic) abnormalities. Patients with SAML are more likely to be older and have other serious medical problems; the blast karyotype is more likely to have complex cytogenetic changes and/or abnormalities of chromosomes 5 and 7 , which have been associated with a poor outcome.

Leukaemic blasts from SAML patients are more often resistant to therapy than those from de novo AML patients. One of the many proposed mechanisms of drug resistance involves the expression of the membrane-associated efflux pumps (transporters) that actively export drugs out of the cell before they can enter the nucleus. ${ }^{4}$ Since all effective cytotoxic regimens interfere with DNA synthesis, this is an important and common cause of drug resistance. One of the most important proteins in this class of transporters is P-glycoprotein (P-gp/MDR-1). ${ }^{4}$ Expression of $P$-gp in the blast cells from elderly patients with AML ranges from 50 to $71 \% .2,5-7$ The anthracyclines, mitoxantrone, etoposide and calicheamicin (the toxin linked to the anti-CD33 monoclonal antibody of gemtuzumab ozogamicin), cytotoxic drugs commonly used for remission induction therapy, are all substrates for this efflux pump. Thus, resistance to one drug usually translates to cross-resistance to all of the others, and has given rise to the concept of multidrug resistance (MDR).

The role of MDR in treatment outcome has been evaluated in several recent clinical studies. In one such study, Southwest Oncology Group

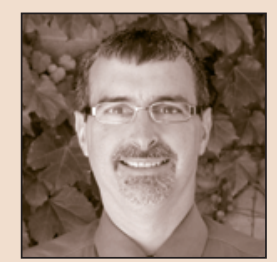

Harry P Erba is an Associate Professor in the Division of Hematology/Oncology at University of Michigan Health Systems and an Executive Officer of the Southwest Oncology Group. He has held Assistant and Associate Physician appointments with the Brigham and Women's Hospital, the Dana-Farber Cancer Institute and Harvard University. Dr Erba's interests include acute and chronic leukaemias and myelodysplastic syndromes. He received his degree in biology from Yale University and his medical and doctoral degree in biophysics from Stanford University in 1988.

E: hperba@med.umich.edu
(SWOG) 9031, the CR rate following standard 3+7 therapy among patients whose leukaemic blasts expressed the P-gp/MDR-1 efflux protein was only $32 \%$, and among the subset with SAML was only $12 \% .{ }^{5}$ In this study the independent variables predictive of response were age, SAML, MDR-1 expression and unfavourable-risk blast karyotype. The decrement in CR rate increased with the coupling of these risk factors in the same patient - no risk factor, $81 \% C R$; 1 factor, 44\% CR; 2 factors, $24 \% C R$; and 3 factors, $12 \% \mathrm{CR}^{2}-$ underscoring the importance of considering clinical risk factors in evaluating therapeutic results in AML. However, attempts to improve the rate of $C R$ through pharmacological inhibition of the P-gP/MDR transporter with cyclosporine, PSC-833 (valspodar) and zosuquidar have been unable to show clinical benefit. ${ }^{7-12}$

\section{Pre-clinical and Phase I Studies of Xanafide}

Xanafide (amonafide L-malate), a novel inhibitor of topoisomerase II (topo II), is an effective drug for the treatment of patients with SAML. Xanafide is an ATP-independent topo II inhibitor that intercalates into DNA. ${ }^{13,14}$ Recent studies in MDR cell lines identified that Xanafide is neither a substrate for nor an inhibitor of $\mathrm{P}-\mathrm{gp}^{15}$ and other MDR efflux transporters associated with drug resistance in $A M L$, such as breast cancer-resistant protein (BCRP) and multidrug-resistant protein-1 (MRP-1). ${ }^{16}$ Xanafide retains cytotoxic activity in cell lines that overexpress P-gp, and consequently are resistant to all of the 'classic' topo II inhibitors used in AML therapy (daunorubicin, doxorubicin, idarubicin, etoposide and mitoxantrone). ${ }^{15,16}$ In one recent survey of leukaemia cells from 22 patients with sAML, P-gp overexpression was present in 60\% and P-gp function (actual efflux) was present in 32\% of the samples. ${ }^{16}$ Half of the samples actively exported daunorubicin out of the cell, while only $9 \%$ actively exported Xanafide. Thus, the poor activity of daunorubicin and the other classic topo II inhibitors in SAML may be due in part to the MDR drug efflux mechanism. In contrast, the encouraging clinical efficacy of Xanafide in SAML (described below) may be attributable in part to its ability to bypass the MDR-associated drug efflux mechanisms commonly expressed in AML cells from poor-risk patients.

Amonafide monotherapy has been tested in clinical trials in acute leukaemia in two phase I studies, and in combination with cytarabine in both phase I and II studies. In the monotherapy trials conducted in patients with refractory AML and other poor risk characteristics, there was substantial dose-related leukaemia cell cytoreduction and $C R$ in patients with poor-risk disease.17,18 The optimal monotherapy dose in both trials was approximately $1,100 \mathrm{mg} / \mathrm{m}^{2} /$ day for five days. The trial reported by Allen et al. enrolled 17 patients, including five with SAML. These five patients had not received prior induction therapy. Two of the five patients with SAML entered CR, lasting for 7.5 and eight months. 
These observations prompted a phase I trial of the combination of amonafide and cytarabine. ${ }^{19}$ The patients were treated with a sevenday continuous infusion of cytarabine $200 \mathrm{mg} / \mathrm{m}^{2} /$ day and a fixed dose of amonafide 600,700 or $800 \mathrm{mg} / \mathrm{m}^{2} /$ day for five days. The most frequent grade 3-4 non-haematological adverse events (AEs) of amonafide at the maximal tolerated dose $\left(700 \mathrm{mg} / \mathrm{m}^{2} /\right.$ day $)$ were emesis $(27 \%)$, rash $(20 \%)$, diarrhoea (13\%) and oedema (13\%). The overall response rate was $46 \%(12 / 26)$, including seven of 15 patients $(47 \%)$ with SAML (see Table 1). Among the seven patients with SAML who achieved $C R$ or good clinical benefit, four received post-remission therapy (three with high-dose cytarabine and one with bone marrow transplantation). These patients sustained a continuous CR for $7.5,8$, $>29$ and >120 months.

Phase II Study of Xanafide in Combination with Cytarabine A subsequent phase II study of Xanafide $600 \mathrm{mg} / \mathrm{m}^{2} /$ day for five consecutive days with cytarabine $200 \mathrm{mg} / \mathrm{m}^{2} /$ day by continuous intravenous infusion for seven days for previously untreated SAML was initiated in September 2005, and completed enrolment in December $2006^{20}$ with follow-up still ongoing. A total of 88 patients were enrolled. Median age was 63 years, $46.6 \%$ male, median performance status 1 (range 0-3) and $47 \%$ with unfavourable-risk blast karyotype. Overall, $45.5 \%$ presented with prior MDS, half of whom received prior therapy for MDS with only one achieving CR of MDS. Fifty-four per cent of patients had tAML, having previously received a median of two (range one to six) chemotherapy \pm radiation regimens for nonleukaemic diseases.

The primary end-point of the trial was $C R$ rate with or without complete haematopoietic recovery (CR plus CRi), which occurred in 37 out of $88(42.0 \%)$ of the patients (see Table 2 ). The CR rate was maintained across different poor-risk subgroups, including older patients ( $\geq 60$ years, $43.6 \%$ ), patients with tAML $(41.7 \%)$ and patients who received prior treatment for their MDS (36.0\%). The CR rate was higher in those with intermediate-risk karyotypes than those with unfavourable-risk karyotypes (60 versus $22 \%$, respectively). The median duration of remission is estimated to be 312 days in this ongoing study. Forty-three per cent of patients are in remission at 12 months. These results compare favourably with the historical experience of patients with SAML who have similar poor-risk features treated with standard $3+7$ therapy as reported by the SWOG, in which CR rates were approximately $25 \% .^{2}$ Six of 10 patients who had abnormal blast cell karyotypes at onset of therapy had reversion to normal karyotypes in the $C R$ marrow assessment following the Xanafide/cytarabine therapy.

The combination of Xanafide plus cytarabine was associated with predictable and manageable toxicities. Only one of 102 Xanafide infusion courses was interrupted due to an $A E$, and only five of the 88 patients $(5.7 \%)$ discontinued due to an AE. The most common non-haematological AEs due to amonafide were nausea $(73.9 \%)$, diarrhoea (51.1\%), emesis (36.4\%) and pyrexia (31.8\%), with the
Table 1: Phase I Trial of the Combination of Xanafide and Cytarabine in Patients with SAML, Relapsed de novo AML and CML Blast Crisis

\begin{tabular}{lccc}
\hline & Secondary AML & Relapsed de novo AML & CML Blast Crisis \\
\hline Number enrolled & 15 & 8 & 3 \\
\hline CR & 5 & 4 & 1 \\
\hline Clinical benefit & 2 & 0 & 0 \\
\hline
\end{tabular}

$A M L$ = acute myeloid leukaemia; $C M L=$ chronic myeloid leukaemia; $C R=$ complete remission. Clinical benefit = complete remission with occasional circulating peripheral blasts for 10 months in one patient, and complete remission for 28 days in second patient.

Table 2: Phase II Trial of the Combination of Xanafide and Cytarabine in Patients with Secondary AML $(n=88)$

\begin{tabular}{lc} 
& Per cent \\
\hline Overall response (CR+CRp) & 42.0 \\
CR & 38.6 \\
CRp & 3.4 \\
\hline Age & \\
$<60$ years & 39.4 \\
$\geq 60$ years & 43.6 \\
\hline Type of SAML & \\
Prior MDS & 42.5 \\
tAML & 41.7 \\
\hline
\end{tabular}

$C R$ = complete remission; $C R p=C R$ with incomplete recovery of the platelet count; $S A M L=$ secondary acute myeloid leukaemia; MDS = myelodysplastic syndrome; tAML = treatment-related $A M L$.

most common grade 3, 4 and 5 events being bacteraemia (7.9\%), hypotension (7.9\%), pneumonia (7.9\%), diarrhoea (6.8\%) and rash (6.8\%). Forty-four patients experienced one or more grade 3 or 4 serious AEs, most of which were related to infectious disease and related complications. Median time to recovery of ANC to $>0.5 \times 109 / 1$ was 28 days, and to platelet count $>20 \times 109 / /$ was 27 days. Eighteen deaths $(20.5 \%)$ occurred within 28 days after the first dose of Xanafide, typically related to infectious disease complications and/or progressive disease.

\section{Summary and Conclusions}

Xanafide is a unique topo II inhibitor that, unlike the classic topo II inhibitors used in the treatment of AML, maintains its cytotoxic effect on leukaemia cells despite overexpression of P-gp and the MDR phenotype. This characteristic circumvents a major cause of drug resistance in poor-risk AML. Treatment of patients with SAML with the combination of Xanafide and cytarabine is associated with an acceptable safety profile. The observed $C R$ rates associated with Xanafide and cytarabine in these patients with SAML compare favourably with published studies of similar patients with SAML treated with the standard $3+7$ regimen: 42 versus $25 \%$, respectively. Xanafide maintains clinical efficacy across multiple poor-risk subgroups, including older patients, patients with tAML and patients with MDS whose disease was refractory to prior MDS therapy, most notably the DNA methyltransferase inhibitors. The combination of Xanafide and cytarabine is currently being investigated in a randomised phase III trial in comparison with the standard regimen of $3+7$.
1. Cheson BD, et al., J Clin Oncol, 2003;21:4642-9.

2. Leith CP, et al., Blood, 1997:89:3323-9.

3. Erba HP, Hematology, 2007:420-28.

4. Willman CL, Semin Hematol, 1997;34:25-33.

5. Godwin JE, et al., Blood, 1998:91:3607-15.

6. Anderson JE, et al., Blood, 2002;100:3869-76.

7. Baer MR, et al., Blood, 2002;100:1224-32.
8. List AF, et al., Blood, 2001;98:3212-20.

9. Kolitz JE, et al., J Clin Oncol, 2004;22:4290-4301.

10. Kolitz JE, et al., Blood, 2005;106: abstract 407.

11. van der Holt B, et al., Blood, 2005;106:2646-54.

12. Cripe $L D$, et al., Blood, 2006:108:129a.

13. Tsiang YH, et al., Mol Pharmacol, 1989;36:371-6.

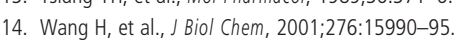

15. Chau M, et al., Leuk Res, 2008;32:465-73.

16. O'Loughlin KL, et al., Blood, 2007;110:702a: abstract 2380.

17. O'Brien S, et al., Cancer Res, 1991; 51:935-8.

18. Allen SL, et al., Proc Am Soc Clin Oncol, 1992;11:276s.

19. Allen SL, et al., Proc Am Soc Clin Oncol, 2005:23:585s.

20. Erba HP, et al., Proc Am Soc Clin Oncol, 2007;25:373s. 